\title{
microRNA-23b regulates the expression of inflammatory factors in vascular endothelial cells during sepsis
}

\author{
MING WU ${ }^{1}$, JIAN-TENG GU ${ }^{1}$, BIN YI $^{1}$, ZHONG-ZHI TANG ${ }^{2}$ and GUO-CAI TAO ${ }^{1}$ \\ ${ }^{1}$ Department of Anesthesiology, Southwest Hospital, Third Military Medical University, Chongqing 400038; \\ ${ }^{2}$ Department of Emergency, Wuhan General Hospital of Guangzhou Military Command, Wuhan, Hubei 430070, P.R. China
}

Received May 11, 2014; Accepted January 7, 2015

DOI: $10.3892 / \mathrm{etm} .2015 .2224$

\begin{abstract}
R-23b is a multifunctional microRNA that contributes to the regulation of multiple signaling pathways. It has been reported that miR-23b prevents multiple autoimmune diseases through the regulation of inflammatory cytokine pathways. In addition, the function and underlying mechanisms of miR-23b on sepsis are currently being investigated. In the present study, miR-23b inhibitor and mimics sequences were transfected into human vascular endothelial cells to inhibit and upregulate the expression of miR-23b, respectively. In addition, respective negative control (NC) sequences were transfected. The expression of miR-23b was found to be downregulated in the cells transfected with the mimics NC or inhibitor NC sequences following stimulation with lipopolysaccharide (LPS; $\mathrm{P}<0.01$ ); however, higher expression levels were maintained in the cells transfected with the mimics sequence and very low levels were observed in the cells transfected with the inhibitor sequence. In addition, the expression levels of nuclear factor (NF) $\kappa \mathrm{B}$, tumor necrosis factor (TNF)- $\alpha$, interleukin (IL)-6, intercellular adhesion molecule (ICAM)-1, E-selectin and vascular cell adhesion molecule (VCAM)-1 were shown to increase following induction by LPS in the cells transfected with inhibitor/mimics NC sequences $(\mathrm{P}<0.05)$. However, the expression levels of these inflammatory factors decreased in the cells transfected with the mimics sequence, and increased to a greater degree in the cells transfected with the inhibitor sequence, as compared with the inhibitor $\mathrm{NC}$ sequences $(\mathrm{P}<0.05)$. Therefore, miR-23b may play a significant role in the pathogenesis and progression of sepsis by inhibiting the expression of inflammatory factors, including NF- $\kappa$ B, TNF- $\alpha$, IL-6, ICAM-1, E-selectin and VCAM-1.
\end{abstract}

Correspondence to: Professor Guo-Cai Tao, Department of Anesthesiology, Southwest Hospital, Third Military Medical University, 38 Gaotanyan Street, Chongqing 400038, P.R. China E-mail: taoguocaichongqing@163.com

Key words: miR-23b, vascular endothelial cell, sepsis, nuclear factor- $\kappa \mathrm{B}$, tumor necrosis factor- $\alpha$, interleukin-6, intercellular adhesion molecule-1, E-selectin, vascular cell adhesion molecule-1

\section{Introduction}

Sepsis is a systemic inflammatory reaction syndrome that is caused by infectious factors, and is one of the major causes of mortality in critical patients. Deregulation of the body during sepsis is considered to result in uncontrolled inflammation, the release of large amounts of inflammatory mediators, the development of inflammatory cascades and ultimately damage to tissues and organs (1-4). Vascular endothelial cells (VECs) serve as a vital interface between the blood and tissues. In the presence of inflammatory stimulation, the cells are activated and express adhesion molecules that play a critical role in leukocyte aggregation (5). Previous studies have shown that VECs are the major victim to pathogens and their toxins in sepsis. For instance, endotoxin and other bacterial components act on VECs to reduce vascular tension, widen the space between the VECs, increase vascular permeability, promote the release of inflammatory mediators and aggravate platelet aggregation (6). As a result, the inflammatory and coagulation systems become deregulated and systemic inflammatory response syndrome and multiple organ dysfunction syndrome develop $(7,8)$. The nuclear factor $(\mathrm{NF})-\kappa \mathrm{B}$ signaling pathway plays an important regulatory role in sepsis $(9,10)$, and blocking the $\mathrm{NF}-\kappa \mathrm{B}$ pathway is an important modality in the treatment of sepsis $(11,12)$.

microRNA (miRNA) is a small, single-stranded RNA molecule that is ubiquitously present in eukaryotic organisms, which is characterized by high conservation and tissue specificity. miRNA binds to specific mRNA molecules to inhibit the expression of target genes or degrade the mRNA, which subsequently contributes to cell proliferation, differentiation, development, metabolism, apoptosis and other physiological activities. Thus, miRNA exerts an important regulatory function on eukaryotic genes (13-15).

miR-23b is a multifunctional miRNA that contributes to the regulation of multiple signaling pathways, affecting cell proliferation, differentiation, apoptosis and adhesion (16-24). Moreover, the functions and underlying mechanisms are currently under investigation. It has been reported that miR-23b prevents multiple autoimmune diseases through the regulation of inflammatory cytokine pathways, in which the molecule regulates a number of inflammatory cytokines, such as $N F-\kappa B$, tumor necrosis factor (TNF)- $\alpha$, interleukin (IL)-1 $\beta$ and IL-17 $(25,26)$. Therefore, it was hypothesized 
that miR-23b may act on sepsis through the $\mathrm{NF}-\kappa \mathrm{B}$ pathway and IL-17; thus, regulating the $\mathrm{NF}-\kappa \mathrm{B}$-mediated activation of VECs.

In the present study, septic VECs were simulated using bacterial lipopolysaccharide (LPS) to induce the activation of human VECs, after which the cells were transfected with miR-23b mimics and inhibitor sequences to observe the effect of upregulating and inhibiting miR-23b on the expression levels of inflammatory factors in septic VECs. The aim of the present study was to investigate the potential of miR-23b as a therapeutic target for sepsis treatment.

\section{Materials and methods}

Cell culture and miR-23b sequences. The 1D3 human VEC cell line (Shanghai Bioleaf Biotech Co., Ltd., Shanghai, China) was preserved in liquid nitrogen in the laboratory. The cells were routinely cultured in modified RPMI-1640 medium containing 10\% fetalbovineserum(FBS;Hyclone;GEHealthcare,Logan,UT, USA). The following sequences were designed and synthesized by Shanghai GenePharma Co., Ltd (Shanghai, China): miR-23b inhibitor sequence, 5'-GGUAAUCCCUGGCAAUGUGAU-3'; miR-23b inhibitor negative control (NC) sequence, 5'-CAGUACUUUUGUGUAGUACAA-3'; miR-23b mimics sequence, 5'-AUCACAUUGCCAGGGAUUACC-3'; miR-23b mimics NC sequence, 5'-UUCUCCGAACGUGUCACGUTT-3'. The sequences were labeled with fluorescein amidite to observe fluorescence.

Transfection of miR-23b into the human VECs. Using Lipofectamine 2000 transfection reagent (Invitrogen Life Technologies, Carlsbad, CA, USA), the synthesized sequences were transfected into the human VECs. Initially, the mimics $\mathrm{NC}$ or inhibitor NC sequences were used to investigate the optimum conditions for transfection. At day one prior to transfection, $1 \times 10^{4}-3 \times 10^{4}$ cells were inoculated into 24 -well plates, and $500 \mu 1$ modified RPMI-1640 medium containing $10 \%$ FBS was added to each well. The cells were cultured in an incubator containing $5 \% \mathrm{CO}_{2}$ at $37^{\circ} \mathrm{C}$ until the cells reached a confluence of 70-90\%. Various doses of mimics NC or inhibitor $\mathrm{NC}(6,15,20,30,50$ or $100 \mathrm{pmol})$ were added to $50 \mu \mathrm{l}$ serum-free Opti-MEM (Hyclone; GE Healthcare), which was followed by gentle mixing. Lipofectamine $2000(0.3$ or $1 \mu \mathrm{l})$ was added to $50 \mu \mathrm{l}$ serum-free Opti-MEM, mixed gently and rested at room temperature for $5 \mathrm{~min}$. The two solutions were subsequently mixed and added to the plate wells containing the cells and $500 \mu \mathrm{l}$ serum-free RPMI-1640 medium, after which the plates were placed onto a swing bed for gentle shaking. Following incubation for $5 \mathrm{~h}$ at $37^{\circ} \mathrm{C}$, the medium was replaced with $500 \mu \mathrm{l}$ fresh modified RPMI-1640 medium containing serum and the plates were swung for mixing. After a further $24 \mathrm{~h}$ incubation at $37^{\circ} \mathrm{C}$, the cells were observed and photographed under fluorescence microscopy (CX41-32RFL; Olympus, Tokyo, Japan). Pilot studies identified the optimal transfection dose of the miR-23b sequence to be $50 \mathrm{pmol}$ and the optimal dose of Lipofectamine 2000 to be $1 \mu 1$, according to fluorescence intensity measurements. Therefore, the dose of the miR-23b inhibitor, NC inhibitor, miR-23b mimics and NC mimics sequences was $50 \mathrm{pmol}$, and $1 \mu 1$ Lipofectamine 2000 was used for transfection. The procedure for transfection was applied as aforementioned. In addition, a blank control group was established by adding the same volume of phosphate-buffered saline and Lipofectamine 2000.

LPS-stimulated human VECs. In total, $2 \times 10^{4}-5 \times 10^{4}$ cells were successfully transfected with miR-23b and inoculated into 12 -well plates. Into each well, $500 \mu 1$ modified RPMI-1640 medium, containing $10 \% \mathrm{FBS}$, was added. The cells were cultured for $24 \mathrm{~h}$ at $37^{\circ} \mathrm{C}$ in an incubator containing $5 \% \mathrm{CO}_{2}$. Upon reaching a confluence of $70-90 \%$, LPS was added to a final concentration of $1 \mu \mathrm{g} / \mathrm{ml}$. The cells were cultured for 4 and $8 \mathrm{~h}$, after which the total RNA was extracted for quantitative polymerase chain reaction (PCR).

Quantitative PCR. Total RNA extraction was performed according to the instructions of the TRIzol reagent kit (Invitrogen Life Technologies). Reverse transcription of the mRNA was conducted using a RevertAid First Strand cDNA Synthesis kit (Thermo Fisher Scientific, Waltham, MA, USA). The reaction system included $2 \mu \mathrm{l}$ RNA and $1 \mu \mathrm{l}$ oligo (dT)18 primer, and the final volume was adjusted to $12 \mu \mathrm{l}$ with RNase-free deionized water. The solution was incubated at $70^{\circ} \mathrm{C}$ for 5 min on a PikoReal PCR amplifier (Thermo Fisher Scientific), and placed on ice for quick cooling. Next, $4 \mu 15 \mathrm{X}$ buffer, $2 \mu 1 \mathrm{dNTP}(10 \mathrm{mM}), 1 \mu \mathrm{l}$ RNA inhibitor and $1 \mu \mathrm{l}$ reverse transcriptase (Thermo Fisher Scientific) were added to the solution, which was followed by mixing using a pipette. The solution was incubated at $42^{\circ} \mathrm{C}$ for $60 \mathrm{~min}$ on a PCR amplifier, followed by $5 \mathrm{~min}$ incubation at $70^{\circ} \mathrm{C}$ to inactivate the reverse transcriptase. The products of the reverse transcription reaction were quantitatively analyzed using THUNDERBIRD ${ }^{\mathrm{TM}}$ $\mathrm{SYBR}^{\circledR}$ qPCR Mix kit (Toyobo Co., Ltd., Osaka, Japan). A 0.2-ml PCR tube was used, which contained $12.5 \mu 12 \mathrm{X}$ qPCR Mix, $2.0 \mu \mathrm{l}$ gene primer or internal standard primer $(2.5 \mu \mathrm{M})$,

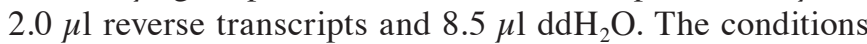
for amplification were as follows: Initial denaturation at $95^{\circ} \mathrm{C}$ for $3 \mathrm{~min}$, followed by 40 cycles of $95^{\circ} \mathrm{C}$ for $15 \mathrm{sec}, 59^{\circ} \mathrm{C}$ for $30 \mathrm{sec}$ and $72^{\circ} \mathrm{C}$ for $25 \mathrm{sec}$. The quantitative PCR assay of miR-23b was performed using a custom reverse transcription and quantitative PCR kit (Changzhou Chutian Biotechnology Co., Ltd., Changzhou, China), where U6 was used as an internal standard, according to the instructions of the kit. Table I shows the sequences of the primers used for quantitative PCR.

Western blot analysis. Cells were harvested by centrifugation at $2,000 \mathrm{x} \mathrm{g}$ for $5 \mathrm{~min}$ at $37^{\circ} \mathrm{C}$, and $10^{6}$ cells were added to $250 \mu 1$ radioimmunoprecipitation assay lysis buffer (Beyotime Institute of Biotechnology, Haimen, China) to extract the total protein. In total, $50 \mu \mathrm{g}$ protein was subjected to SDS-PAGE, after which the protein was transferred onto a $0.45-\mu \mathrm{m}$ polyvinylidene fluoride membrane (Millipore Corporation, Billerica, MA, USA). The membrane was incubated with mouse monoclonal NF-кB P65 (1:1,000; cat. no. 13346S, Cell Signaling Technology, Inc., Danvers, MA, USA), rabbit monoclonal TNF- $\alpha$ (1:1,000; cat. no. ab53450), rabbit monoclonal IL-6 (1:1,000; cat. no. ab32530), rabbit monoclonal VCAM-1 (1:1,000; cat. no. ab134047, Abcam, Cambridge, UK), rabbit polyclonal ICAM-1 (1:1,000; cat. no. sc-7891), rabbit polyclonal E-selectin $(1: 1,000$; cat. no. sc-14011) and rabbit polyclonal $\beta$-actin antibodies $(1: 1,000$; cat. 
Table I. Quantitative polymerase chain reaction primers.

\begin{tabular}{|c|c|c|}
\hline Gene & Primers $\left(5^{\prime} \rightarrow 3^{\prime}\right)$ & Length (bp) \\
\hline NF-кB (NM_003998,NM_001165412) & $\begin{array}{l}\text { Sense, TGAGTCCTGCTCCTTCCAA } \\
\text { Antisense, GAGAGGTGGTCTTCACTGGG }\end{array}$ & 150 \\
\hline IL-6 (NM_000600) & $\begin{array}{l}\text { Sense, AAGCAGCAAAGAGGCACTG } \\
\text { Antisense, TTTCACCAGGCAAGTCTCCT }\end{array}$ & 106 \\
\hline TNF- $\alpha$ (NM_000594) & $\begin{array}{l}\text { Sense, GTGCTGGCAACCACTAAGAAT } \\
\text { Antisense, GCCTAAGGTCCACTTGTGTCA }\end{array}$ & 170 \\
\hline VCAM-1(NM_001078) & $\begin{array}{l}\text { Sense, GCTGCTCAGATTGGAGACTCA } \\
\text { Antisense, CGCTCAGAGGGCTGTCTATC }\end{array}$ & 100 \\
\hline E-selectin (NM_000450) & $\begin{array}{l}\text { Sense, AATCCAGCCAATGGGTTCG } \\
\text { Antisense, GCTCCCATTAGTTCAAATCCTTCT }\end{array}$ & 104 \\
\hline ICAM-1 (NM_000201) & $\begin{array}{l}\text { Sense, GCTCAAGTGTCTAAAGGATGGC } \\
\text { Antisense, CATTATGACTGCGGCTGCTA }\end{array}$ & 196 \\
\hline
\end{tabular}

NF, nuclear factor; IL, interleukin; TNF, tumor necrosis factor; ICAM, intercellular adhesion molecule; VCAM, vascular cell adhesion molecule.

no. sc-130656, Santa Cruz Biotechnology, Inc., Santa Cruz, CA, USA), and shaken overnight at $4^{\circ} \mathrm{C}$. Following discoloration, a horseradish peroxidase-conjugated goat anti-rabbit or goat anti-mouse secondary antibody (1:5,000; KPL, Inc., Gaithersburg, MD, USA) was added and incubated at room temperature for $30 \mathrm{~min}$ to wash out the unbound antibodies. Color development was performed by adding an enhanced chemiluminescence developer (Pierce Biotechnology, Inc., Rockford, IL, USA) and waiting for 1-2 min. Thereafter, the remnant liquid was discarded, and the membrane was embedded with a fresh membrane and subjected to X-ray exposure. The exposure condition was adjusted according to the light intensity. Following development and fixation, the images were analyzed for grayscale using BandScan 5.0 software (Glyko, Novato, CA, USA).

Statistical analysis. All experiments were performed three times, and data are expressed as the mean \pm standard deviation. A $\chi^{2}$ or two-sided t-test was performed for statistical analysis using SPSS 16.0 software (SPSS, Inc., Chicago, IL, USA). $\mathrm{P}<0.05$ was considered to indicate a statistically significant difference.

\section{Results}

Transfection of the miR-23b inhibitor sequence inhibits miR-23b expression, while transfection of the miR-23b mimics sequence increases miR-23b expression in human $V E C s$. Human VECs transfected with miR-23b emitted green fluorescence under fluorescence microscopy (Fig. 1A). The quantitative PCR results indicated that miR-23b expression decreased significantly in the group transfected with the miR-23b inhibitor sequence when compared with the blank control group and the group transfected with the inhibitor NC sequence, demonstrating that transfection with the miR-23b inhibitor inhibited miR-23b expression effectively. By contrast, miR-23b expression increased significantly in the group transfected with the miR-23b mimics sequence when compared with the blank control group and the mimics NC group, indicating that transfection with miR-23b mimics promoted miR-23b expression (Fig. 1B and C).

LPS downregulates miR-23b expression in human VECs. VECs in sepsis were simulated using LPS-stimulated human VECs. The results demonstrated that miR-23b expression decreased significantly in the cells transfected with the mimics NC or inhibitor NC sequences at 4 or $8 \mathrm{~h}$ after LPS stimulation when compared with the cells that did not undergo LPS stimulation $(\mathrm{P}<0.01)$. In addition, the results revealed that $\mathrm{miR}-23 \mathrm{~b}$ expression decreased in the LPS-stimulated human VECs; thus, VEC activation in sepsis was accompanied by the inhibition of miR-23b expression. By contrast, miR-23b expression decreased slightly in the cells transfected with the mimics sequence at 4 or $8 \mathrm{~h}$ after LPS stimulation when compared with the unstimulated cells, but remained at a high level. miR-23b expression remained low in the cells transfected with the inhibitor sequence following LPS stimulation (Fig. 2).

LPS promotes inflammatory cytokine expression in human $V E C s$. Expression levels of inflammatory cytokines, including NF- $\kappa$ B, TNF- $\alpha$, IL-6, ICAM-1, E-selectin and VCAM-1, increased significantly at 4 and $8 \mathrm{~h}$ after LPS stimulation in the human VECs $(\mathrm{P}<0.05$, Fig. 3). These results demonstrated that LPS stimulated the human VECs to express inflammatory cytokines; thus, contributing to the inflammatory reaction in sepsis.

miR-23b mimics inhibits the LPS-stimulated expression of inflammatory factors. The mRNA expression levels of $\mathrm{NF}-\kappa \mathrm{B}$, TNF- $\alpha$, IL-6, ICAM-1, E-selectin and VCAM-1 increased significantly at 4 and $8 \mathrm{~h}$ after LPS stimulation in the human VECs transfected with the mimics NC sequence $(\mathrm{P}<0.05)$; however, the expression levels decreased significantly in the cells transfected with the mimics sequence ( $\mathrm{P}<0.05$; Fig. 4A). Western blot analysis revealed that the protein expression 
A

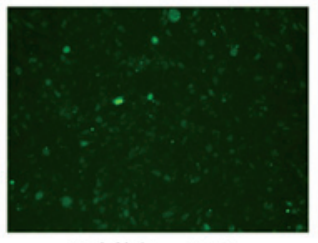

Inhibitor NC

B

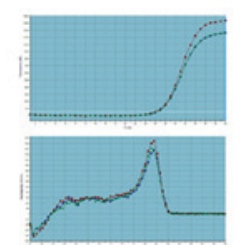

Blank

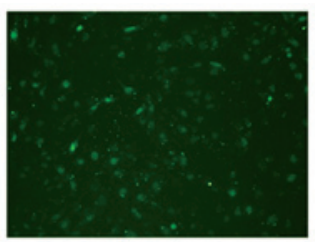

Inhibitor
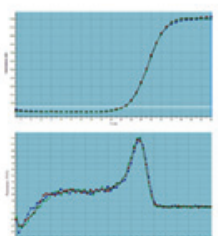

Inhibitor NC

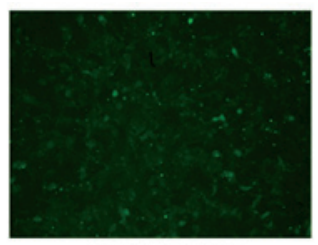

Mimic NC

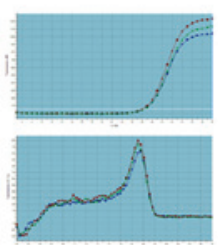

Inhibitor

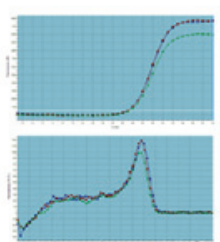

Mimic NC

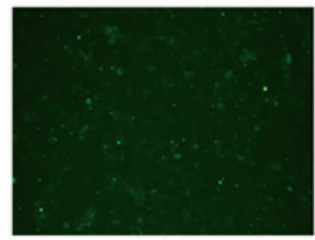

Mimic

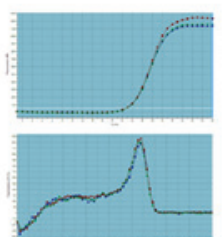

Mimic

$\mathbf{C}$

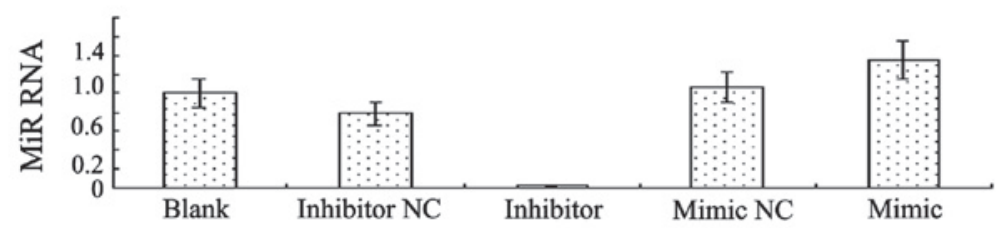

Figure 1. Transfection with the miR-23b inhibitor sequence inhibited miR-23b expression, while transfection with the miR-23b mimics sequence increased miR-23b expression in the human vascular endothelial cells (VECs). (A) Under fluorescence microscopy, the human VECs transfected with miR-23b emitted green fluorescence (magnification, x100). (B and C) Fluorescence quantitative polymerase chain reaction assays revealed that the miR-23b inhibitor sequence significantly inhibited miR-23b expression; however, the miR-23b mimics sequence promoted miR-23b expression. NC, negative control.

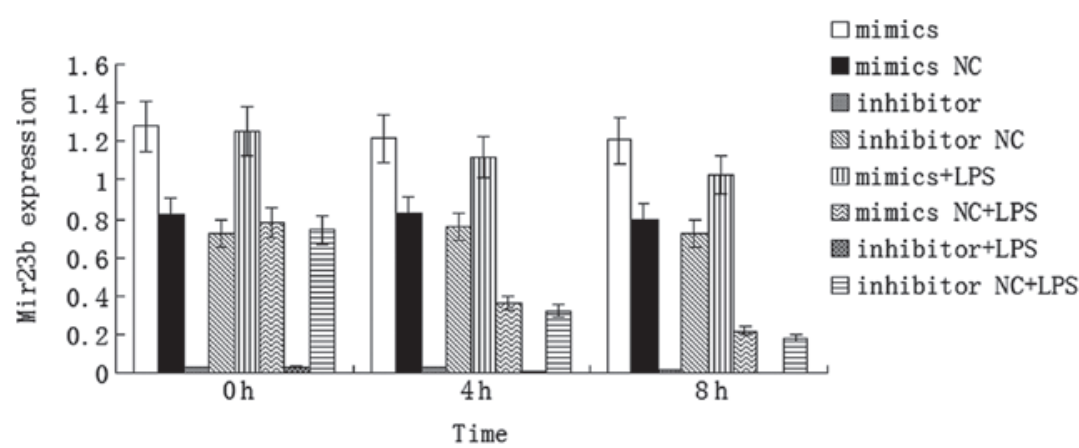

Figure 2. miR-23b expression decreased significantly in the human vascular endothelial cells (VECs) transfected with the mimics NC or inhibitor NC sequences at 4 or $8 \mathrm{~h}$ after LPS stimulation when compared with the human VECs not stimulated with LPS (P<0.01). miR-23b expression remained high in the human VECs transfected with the mimics sequence following LPS stimulation and remained very low in the cells transfected with the inhibitor sequence following LPS stimulation. NC, negative control; LPS, lipopolysaccharide.

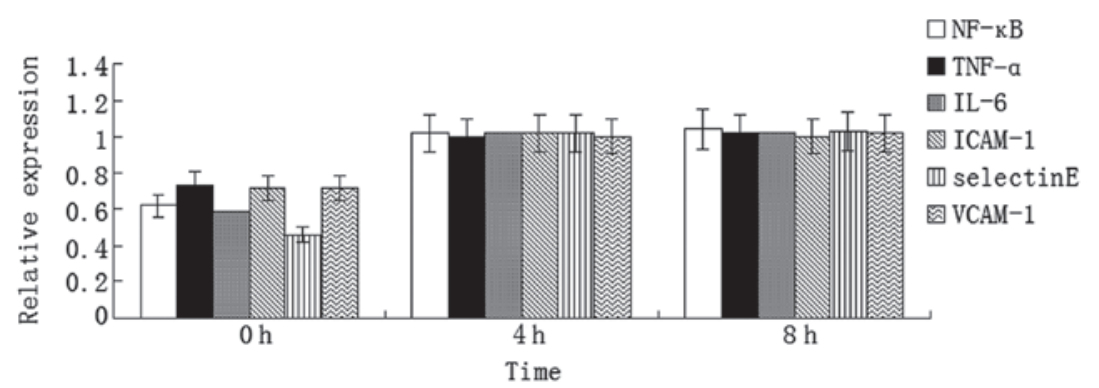

Figure 3. mRNA expression levels of the inflammatory factors increased significantly in the human vascular endothelial cells after 4 and $8 \mathrm{~h}$ of LPS stimulation $(\mathrm{P}<0.05)$. NF, nuclear factor; TNF, tumor necrosis factor; IL, interleukin; ICAM, intercellular adhesion molecule; VCAM, vascular cell adhesion molecule.

levels of NF- $\kappa$ B, TNF- $\alpha$, IL-6, ICAM-1 and E-selectin were significantly lower in the mimics group when compared with the mimics NC group after $4 \mathrm{~h}$ of LPS stimulation $(\mathrm{P}<0.05)$, while the expression level of VCAM-1 was significantly lower in the mimics group when compared with the mimics NC group following $8 \mathrm{~h}$ of LPS stimulation ( $\mathrm{P}<0.05$; Fig. 4B). 
A

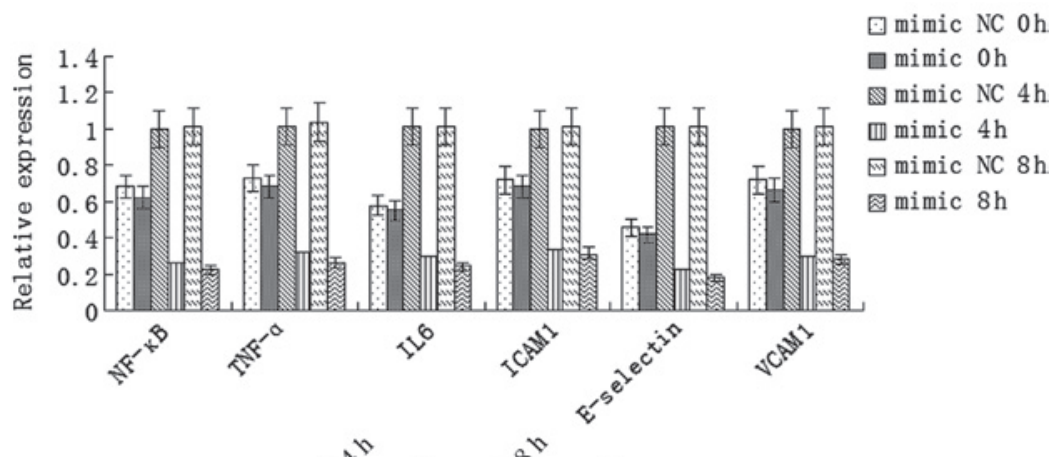

B

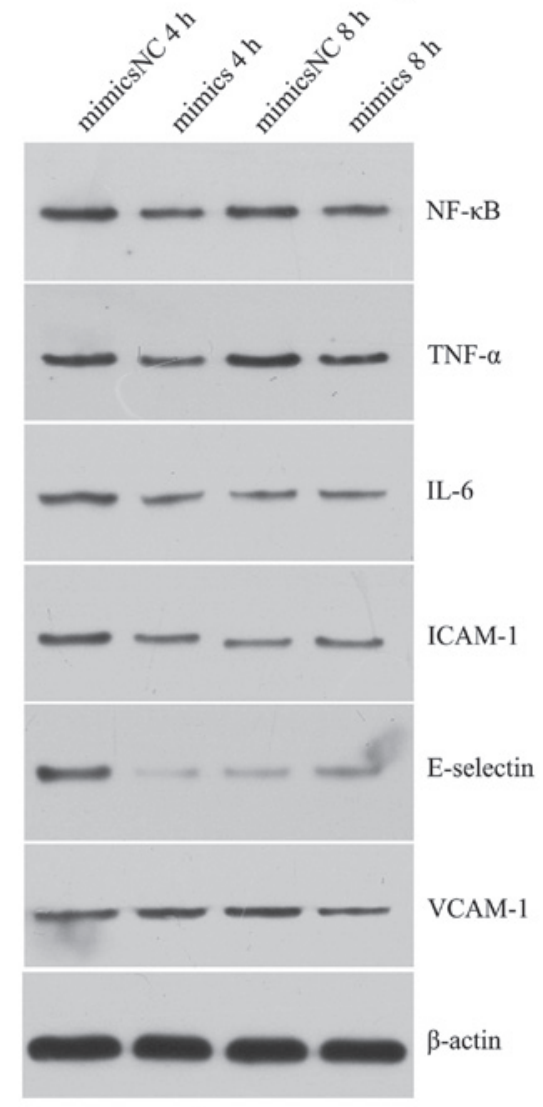

Figure 4. Transfection with the miR-23b mimics sequence inhibited the lipopolysaccharide (LPS)-stimulated expression of inflammatory factors. (A) Quantitative polymerase chain reaction revealed that mRNA expression levels of NF- $\kappa$ B, TNF- $\alpha$, IL-6, ICAM-1, E-selectin and VCAM-1 increased significantly in the human vascular endothelial cells transfected with the mimics NC after 4 and 8 h of LPS stimulation, and decreased significantly in the cells transfected with the mimics sequence $(\mathrm{P}<0.05)$. (B) Western blot analysis revealed that the protein expression levels of NF- $\kappa \mathrm{B}$, TNF- $\alpha$, IL-6, ICAM-1 and E-selectin were significantly lower in the mimics group when compared with the mimics NC group after 4 h of LPS stimulation (P<0.05). Furthermore, the protein expression level of VCAM-1 was significantly lower in the mimics group when compared with the mimics NC group after 8 h of LPS stimulation $(\mathrm{P}<0.05)$. NC, negative control; NF, nuclear factor; TNF, tumor necrosis factor; IL, interleukin; ICAM, intercellular adhesion molecule; VCAM, vascular cell adhesion molecule.

Effect of the miR-23b inhibitor sequence on the expression of inflammatory factors in LPS-stimulated cells. The mRNA expression levels of NF- $\kappa \mathrm{B}, \mathrm{TNF}-\alpha$, IL-6, ICAM-1, E-selectin and VCAM-1 increased significantly in the human VECs transfected with the inhibitor NC sequence following LPS stimulation for 4 and $8 \mathrm{~h}$ when compared with the prestimulation levels $(\mathrm{P}<0.05)$. In addition, the expression levels of the inflammatory factors increased significantly in the cells transfected with the inhibitor sequence following LPS stimulation, as compared with the prestimulation levels $(\mathrm{P}<0.05)$. Furthermore, the levels of the inflammatory factors increased significantly after 4 or $8 \mathrm{~h}$ of LPS stimulation when compared with the levels in the cells transfected with the inhibitor NC sequence (Fig. 5A). Western blot analysis revealed that the protein expression levels of NF- $\kappa \mathrm{B}, \mathrm{TNF}-\alpha$, IL-6, ICAM-1, E-selectin and VCAM-1 increased significantly in the cells transfected with the inhibitor sequence when compared with the cells transfected with the inhibitor $\mathrm{NC}$ sequence after 4 or $8 \mathrm{~h}$ of LPS stimulation $(\mathrm{P}<0.05$; Fig. 5B).

\section{Discussion}

Sepsis is a systemic inflammatory reaction syndrome caused by infection, which may lead to shock and multiple organ dysfunction (27). The pathogenesis of sepsis is extremely complex (28). The invasion of pathogens triggers the release of inflammatory factors, which results in systemic inflam- 
A

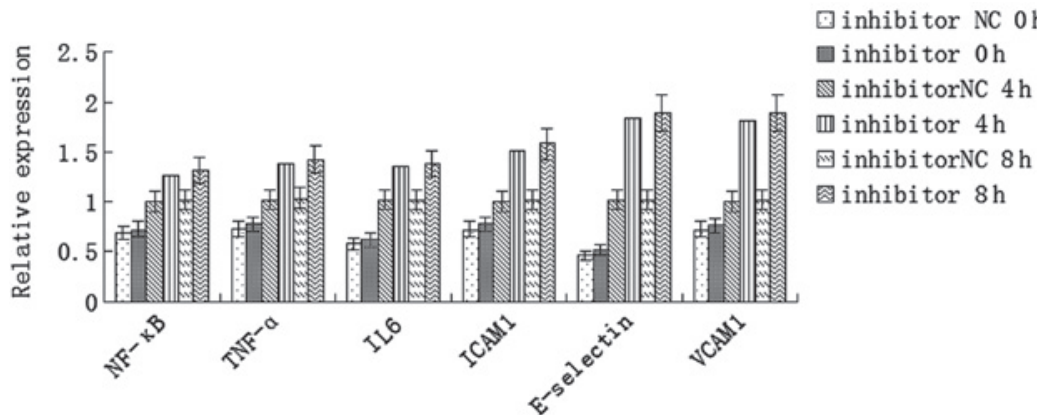

B

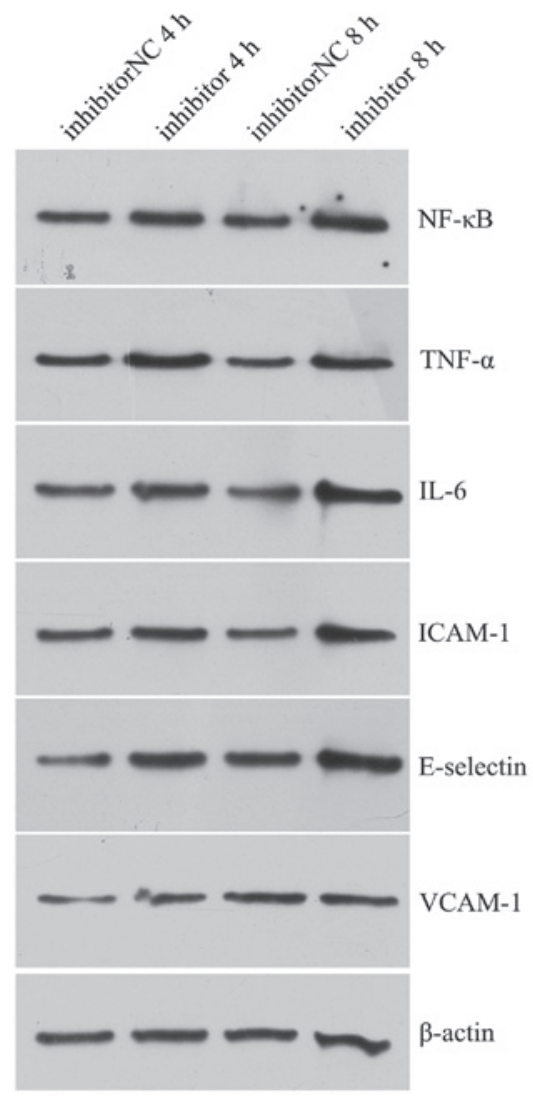

Figure 5. Transfection with the miR-23b inhibitor sequence promoted the expression of the inflammatory factors in lipopolysaccharide (LPS)-stimulated cells. (A) mRNA expression levels of NF- $\mathrm{B}$, TNF- $\alpha$, IL-6, ICAM-1, E-selectin and VCAM-1 increased significantly in the human vascular endothelial cells transfected with the inhibitor NC after 4 and $8 \mathrm{~h}$ of LPS stimulation when compared with the prestimulation levels (P<0.05). In addition, expression levels of these factors increased significantly in the cells transfected with the miR-23b inhibitor sequence following LPS stimulation when compared with the prestimulation levels $(\mathrm{P}<0.05)$. The expression levels of these factors were greater in the cells transfected with miR-23b inhibiter as compared with those transfected with the inhibitor NC after 4 or $8 \mathrm{~h}$ of LPS stimulation. (B) Western blot analysis revealed that the protein expression levels of NF- $\kappa$ B, TNF- $\alpha$, IL-6, ICAM-1, E-selectin and VCAM-1 increased significantly after 4 or $8 \mathrm{~h}$ of LPS stimulation in the cells transfected with the inhibitor sequence when compared with the cells transfected with the inhibitor $\mathrm{NC}(\mathrm{P}<0.05)$. NC, negative control; NF, nuclear factor; TNF, tumor necrosis factor; IL, interleukin; ICAM, intercellular adhesion molecule; VCAM, vascular cell adhesion molecule.

matory reactions. Currently, the role of miRNA in sepsis has become a focus of research. miRNA, a type of endogenous, non-encoding, single-stranded RNA with a length of 22 nucleotides, can degrade mRNA or inhibit translation, subsequently regulating gene expression at the post-transcriptional level (29). There are a number of target genes of miRNA, with evidence demonstrating that miRNA regulates the expression of $\geq 30 \%$ of human genes $(30,31)$. Upon invasion of pathogenic microorganisms, host cells have been demonstrated to produce miRNA quickly, promoting the release of inflammatory factors to cause immune hyperactivity, and inducing apoptosis or degrading the inflammatory factors to cause immunosuppression (32-37). Therefore, miRNA plays a critical role in regulating inflammatory reactions in sepsis (38). VECs are single-layer squamous cells that cover the vascular lining. The cells serve as a barrier, and are involved in substance exchange, vascular tension regulation, coagulation and the inflammatory reaction. VEC damage is closely associated with sepsis. Pathogenic microorganisms and their products may impair VECs and lead to microcirculation disturbance (39) and the amplification of inflammatory reactions $(40,41)$. In the present study, LPS-stimulated human VECs were used to simulate VECs in sepsis. LPS is the major cell wall component of Gram-negative bacilli; thus, LPS is a major pathogenic substance that causes toxic reactions. Therefore, LPS is often used to reproduce sepsis models (42). 
The results demonstrated that LPS promoted the human VECs to express various inflammatory factors, including $\mathrm{NF}-\kappa \mathrm{B}$, TNF- $\alpha$, IL-6, ICAM-1, E-selectin and VCAM-1 (Fig. 2).

miR-23b is a newly identified miRNA. The molecule has been shown to exert a similar function to an oncogene in glioma, urinary system tumors (kidney cancer and prostatic carcinoma) and breast cancer (43-48). miR-23b regulates cytoskeletal reconstruction, cell invasion and metastasis (49). In addition, miR-23b has been shown to inhibit inflammatory reactions in autoimmune diseases; thus, can exert protection against autoimmune diseases $(50,51)$. However, to the best of our knowledge, there have been no previous studies investigating the role of miR-23b in sepsis. The present study demonstrated that miR-23b expression decreased in LPS-stimulated human VECs. In order to investigate the regulatory mechanisms of miR-23b on inflammatory factor expression in VECs during sepsis, miR-23b expression in human VECs was upregulated and downregulated through transfection with miR-23b mimics and inhibitor sequences, as well as the respective NC sequences. The results demonstrated that upregulation of miR-23b inhibited the expression of NF- $\kappa$ B, TNF- $\alpha$, IL-6, ICAM-1, E-selectin and VCAM-1, while downregulation of miR-23b promoted inflammatory factor expression.

Numerous inflammatory factors interact with each other to form a complex network, which is critical to sepsis $(52,53)$. Previously, NF- $\kappa \mathrm{B}$ has been shown to be activated in sepsis, regulating apoptosis, cell growth, the stress reaction, the immune reaction and septic shock (54-56). In addition, the levels of TNF- $\alpha$ and IL- 6 have been shown to reach a peak as early as $3 \mathrm{~h}$ after the onset of sepsis, and the degree of increase may reflect the severity of sepsis $(57,58)$. ICAM-1, VCAM-1 and E-selectin are important cell adhesion factors that regulate the activity of inflammatory and vascular endothelial cells, pro- and anti-inflammatory factors, as well as inflammatory cell migration to tissues and organs; thus, these factors play an important role in sepsis (59-61).

In conclusion, the present study demonstrated that miR-23b regulates sepsis through inhibiting the expression of inflammatory factors in VECs. The affected inflammatory factors include NF- $\kappa$ B, TNF- $\alpha$, IL-6, ICAM-1, E-selectin and VCAM-1. These results suggest that miR-23b may be a potential therapeutic target for the treatment of sepsis. However, the present study only observed the regulation of inflammatory factors by miR-23b, however the underlying mechanisms require further study.

\section{References}

1. Dirkes S: Sepsis and inflammation: impact on acute kidney injury. Nephrol Nurs J 40: 125-133, 2013.

2. Gomez H, Ince C, De Backer D, et al: A unified theory of sepsis-induced acute kidney injury: inflammation, microcirculatory dysfunction, bioenergetics and the tubular cell adaptation to injury. Shock 41: 3-11, 2014.

3. Pinheiro da Silva F, Machado MC and Velasco IT: Neuropeptides in sepsis: from brain pathology to systemic inflammation. Peptides 44: 135-138, 2013.

4. Lewis DH, Chan DL, Pinheiro D, Armitage-Chan E and Garden OA: The immunopathology of sepsis: pathogen recognition, systemic inflammation, the compensatory anti-inflammatory response and regulatory $\mathrm{T}$ cells. J Vet Intern Med 26: 457-482, 2012.
5. Hernandez MR, Palomo M,Fuste B, et al: Effect of two different dialysis membranes on leukocyte adhesion and aggregation. Nephron Clin Pract 106: c1-c8, 2007.

6. Rodella LF, Favero G, Foglio E, et al: Vascular endothelial cells and dysfunctions: role of melatonin. Front Biosci (Elite Ed) 5:119-129, 2013.

7. Zhang ZD and Ma XC: Injury of vascular endothelial cell and microcirculation disturbance in sepsis. Zhongguo Wei Zhong Bing Ji Jiu Yi Xue 23: 125-128, 2011 (In Chinese).

8. Li M and Yu YH: Vascular endothelial cell dysfunction and its clinical strategy in severe sepsis. Zhonghua Er Ke Za Zhi 49: 603-606, 2011 (In Chinese).

9. Adamzik M, Schäfer S, Frey UH, et al: The NFKB1 promoter polymorphism (-94ins/delATTG) alters nuclear translocation of NF- $\kappa \mathrm{B} 1$ in monocytes after lipopolysaccharide stimulation and is associated with increased mortality in sepsis. Anesthesiology 118: 123-133, 2013.

10. Raspé C, Höcherl K, Rath S, Sauvant C and Bucher M NF- $\mathrm{KB}$-mediated inverse regulation of fractalkine and CX3CR1 during CLP-induced sepsis. Cytokine 61: 97-103, 2013.

11. Kaplan J, Nowell M, Chima R and Zingarelli B: Pioglitazone reduces inflammation through inhibition of NF- $\kappa \mathrm{B}$ in polymicrobial sepsis. Innate Immun 20: 519-528, 2013

12. Li H, Han W, Polosukhin V, et al: NF- $\mathrm{BB}$ inhibition after cecal ligation and puncture reduces sepsis-associated lung injury without altering bacterial host defense. Mediators Inflamm 2013: 503213, 2013

13. Lenkala D, LaCroix B, Gamazon ER, Geeleher P, Im HK and Huang RS: The impact of microRNA expression on cellular proliferation. Hum Genet 133: 931-938, 2014.

14. Dvinge H, Git A, Gräf S, et al: The shaping and functional consequences of the microRNA landscape in breast cancer. Nature 497: 378-382, 2013.

15. Melton C, Judson RL and Blelloch R: Opposing microRNA families regulate self-renewal in mouse embryonic stem cells. Nature 463: 621-626, 2010.

16. Li W, Liu Z, Chen L, Zhou L and Yao Y: MicroRNA-23b is an independent prognostic marker and suppresses ovarian cancer progression by targeting runt-related transcription factor-2. FEBS Lett 588: 1608-1615, 2014

17. Pellegrino L, Krell J, Roca-Alonso L, Stebbing J and Castellano L: MicroRNA-23b regulates cellular architecture and impairs motogenic and invasive phenotypes during cancer progression. Bioarchitecture 3: 119-124, 2013.

18. Donadelli M and Palmieri M: Roles for microRNA 23b in regulating autophagy and development of pancreatic adenocarcinoma. Gastroenterology 145: 936-938, 2013.

19. Ishteiwy RA, Ward TM, Dykxhoorn DM and Burnstein KL: The microRNA -23b/-27b cluster suppresses the metastatic phenotype of castration-resistant prostate cancer cells. PLoS One 7: e52106, 2012.

20. Au Yeung CL, Tsang TY, Yau PL and Kwok TT: Human papillomavirus type 16 E6 induces cervical cancer cell migration through the $\mathrm{p} 53 /$ microRNA-23b/urokinase-type plasminogen activator pathway. Oncogene 30: 2401-2410, 2011.

21. Wang KC, Garmire LX, Young A, et al: Role of microRNA-23b in flow-regulation of $\mathrm{Rb}$ phosphorylation and endothelial cell growth. Proc Natl Acad Sci USA 107: 3234-3239, 2010.

22. Pellegrino L, Stebbing J, Braga VM, et al: miR-23b regulates cytoskeletal remodeling, motility and metastasis by directly targeting multiple transcripts. Nucleic Acids Res 41: 5400-5412, 2013.

23. Loftus JC, Ross JT, Paquette KM, et al: miRNA expression profiling in migrating glioblastoma cells: regulation of cell migration and invasion by miR-23b via targeting of Pyk2. PLoS One 7: e39818, 2012.

24. Chen L, Han L, Zhang K, et al: VHL regulates the effects of miR-23b on glioma survival and invasion via suppression of HIF-1 $\alpha /$ VEGF and $\beta$-catenin/Tcf- 4 signaling. Neuro Oncol 14: 1026-1036, 2012.

25. Zhu S, Pan W, Song X, et al: The microRNA miR-23b suppresses IL-17-associated autoimmune inflammation by targeting TAB2, TAB3 and IKK- $\alpha$. Nat Med 18: 1077-1086, 2012.

26. Hu R and O'Connell RM: MiR-23b is a safeguard against autoimmunity. Nat Med 18: 1009-1010, 2012.

27. Duran-Bedolla J, Montes de Oca-Sandval MA, Saldaña-Navor V, et al: Sepsis, mitochondrial failure and multiple organ dysfunction. Clin Invest Med 37: E58-E69, 2014

28. Stearns-Kurosawa DJ, Osuchowski MF, Valentine C, Kurosawa S and Remick DG: The pathogenesis of sepsis. Annu Rev Pathol 6: 19-48, 2011. 
29. Brümmer A and Hausser J: MicroRNA binding sites in the coding region of mRNAs: extending the repertoire of post-transcriptional gene regulation. Bioessays 36: 617-626, 2014.

30. Tarang S and Weston MD: Macros in microRNA target identification: a comparative analysis of in silico, in vitro and in vivo approaches to microRNA target identification. RNA Biol 11: 324-333, 2014.

31. Bartel DP: MicroRNAs: genomics, biogenesis, mechanism and function. Cell 116: 281-297, 2004.

32. Jernås M, Malmeström C, Axelsson M, et al: MicroRNA regulate immune pathways in T-cells in multiple sclerosis (MS). BMC Immunol 14: 32, 2013.

33. Li YC, Chen Y, Liu W and Thadhani R: MicroRNA-mediated mechanism of vitamin D regulation of innate immune response. J Steroid Biochem Mol Biol 144: 81-86, 2014.

34. Chen CZ, Schaffert S, Fragoso R and Loh C: Regulation of immune responses and tolerance: the microRNA perspective. Immunol Rev 253: 112-128, 2013.

35. Lourenço AP, Guidugli-Lazzarini KR, Freitas FC, Bitondi MM and Simões ZL: Bacterial infection activates the immune system response and dysregulates microRNA expression in honey bees. Insect Biochem Mol Biol 43: 474-482, 2013.

36. Jernås M, Nookaew I, Wadenvik $\mathrm{H}$ and Olsson B: MicroRNA regulate immunological pathways in T-cells in immune thrombocytopenia (ITP). Blood 121: 2095-2098, 2013.

37. Zhou R, O'Hara SP and Chen XM: MicroRNA regulation of innate immune responses in epithelial cells. Cell Mol Immunol 8: 371-379, 2011.

38. Du LL and Ma ZF: MicroRNA and immune response, and sepsis. Zhongguo Wei Zhong Bing Ji Jiu Yi Xue 21: 501-503, 2009 (In Chinese).

39. Zhang ZD and Ma XC: Injury of vascular endothelial cell and microcirculation disturbance in sepsis. Zhongguo Wei Zhong Bing Ji Jiu Yi Xue 23: 125-128, 2011 (In Chinese).

40. Li M and Yu YH: Vascular endothelial cell dysfunction and its clinical strategy in severe sepsis. Zhonghua Er Ke Za Zhi 49 603-606, 2011 (In Chinese).

41. Herwig MC, Tsokos M, Hermanns MI, Kirkpatrick CJ and Müller AM: Vascular endothelial cadherin expression in lung specimens of patients with sepsis-induced acute respiratory distress syndrome and endothelial cell cultures. Pathobiology 80 : 245-251, 2013.

42. Anand AR, Bradley R and Ganju RK: LPS-induced MCP-1 expression in human microvascular endothelial cells is mediated by the tyrosine kinase, Pyk2 via the p38 MAPK/NF-kappaB-dependent pathway. Mol Immunol 46: 962-968, 2009.

43. Chen L, Zhang K, Shi Z, et al: A lentivirus-mediated miR-23b sponge diminishes the malignant phenotype of glioma cells in vitro and in vivo. Oncol Rep 31: 1573-1580, 2014.

44. Zaman MS, Thamminana S, Shahryari V, et al: Inhibition of PTEN gene expression by oncogenic miR-23b-3p in renal cancer. PLoS One 7: e50203, 2012.

45. Geng J, Luo H, Pu Y, et al: Methylation mediated silencing of miR-23b expression and its role in glioma stem cells. Neurosci Lett 528: 185-189, 2012.
46. Leone V, Langella C, D'Angelo D, et al: MiR-23b and miR-130b expression is downregulated in pituitary adenomas. Mol Cell Endocrinol 390: 1-7, 2014

47. Majid S, Dar AA, Saini S, et al: mir-23b represses proto-oncogene Src kinase and functions as methylation-silenced tumor suppressor with diagnostic and prognostic significance in prostate cancer. Cancer Res 72: 6435-6446, 2012.

48. Jin L, Wessely O, Marcusson EG, et al: Prooncogenic factors miR-23b and miR-27b are regulated by Her2/Neu, EGF and TNF- $\alpha$ in breast cancer. Cancer Res 73: 2884-2896, 2013.

49. Pellegrino L, Stebbing J, Braga VM, et al: miR-23b regulates cytoskeletal remodeling, motility and metastasis by directly targeting multiple transcripts. Nucleic Acids Res 41: 5400-5412, 2013.

50. Hu R and O'Connell RM: MiR-23b is a safeguard against autoimmunity. Nat Med 18: 1009-1010, 2012.

51. Ouda R, Onomoto K, Takahasi K, et al: Retinoic acid-inducible gene I-inducible miR-23b inhibits infections by minor group rhinoviruses through down-regulation of the very low density lipoprotein receptor. J Biol Chem 286: 26210-26219, 2011.

52. Wu Y, Li C, He Y, et al: Relationship between expression of microRNA and inflammatory cytokines plasma level in pediatric patients with sepsis. Zhonghua Er Ke Za Zhi 52: 28-33, 2014 (In Chinese).

53. Chen W, Zhao L, Niu S, et al: The diagnostic value of different pro-inflammatory factor in early diagnosis of sepsis in patients with bloodstream infection. Zhonghua Wei Zhong Bing Ji Jiu Yi Xue 26: 165-170, 2014 (In Chinese).

54. Brown MA and Jones WK: NF-kappaB action in sepsis: the innate immune system and the heart. Front Biosci 9: 1201-1217, 2004.

55. Thair S and Russell JA: Noncanonical nuclear factor kappaB $(\mathrm{NF}-\mathrm{\kappa B})$ signaling and potential for therapeutics in sepsis. Curr Infect Dis Rep 15: 364-371, 2013.

56. Liang Y, Li X, Zhang X, et al: Elevated levels of plasma TNF- $\alpha$ are associated with microvascular endothelial dysfunction in patients with sepsis through activating the NF-kB and p38 mitogen-activated protein kinase in endothelial cells. Shock 41: 275-281, 2014.

57. Song R, Kim J, Yu D, Park C and Park J: Kinetics of IL-6 and TNF- $\alpha$ changes in a canine model of sepsis induced by endotoxin. Vet Immunol Immunopathol 146: 143-149, 2012.

58. Shahkar L, Keshtkar A, Mirfazeli A, Ahani A and Roshandel G: The role of IL- 6 for predicting neonatal sepsis: a systematic review and meta-analysis. Iran J Pediatr 21: 411-417, 2011.

59. Hildebrand F, Pape HC, Harwood P, et al: Role of adhesion molecule ICAM in the pathogenesis of polymicrobial sepsis. Exp Toxicol Pathol 56: 281-290, 2005.

60. Figueras-Aloy J, Gómez-López L, Rodríguez-Miguélez JM, et al: Serum soluble ICAM-1, VCAM-1, L-selectin, and P-selectin levels as markers of infection and their relation to clinical severity in neonatal sepsis. Am J Perinatol 24: 331-338, 2007.

61. Zaki Mel-S and el-Sayed H: Evaluation of microbiologic and hematologic parameters and E-selectin as early predictors for outcome of neonatal sepsis. Arch Pathol Lab Med 133: 1291-1296, 2009. 